\title{
The Sum and Chain Rules for Maximal Monotone Operators
}

\author{
M.D. VOISEI \\ The University of Texas - Pan American, Department of Mathematics, Edin- \\ burg, Texas - 78541, USA
}

\begin{abstract}
This paper is primarily concerned with the problem of maximality for the sum $A+B$ and composition $L^{*} M L$ in non-reflexive Banach space settings under qualifications constraints involving the domains of $A, B, M$. Here $X, Y$ are Banach spaces with duals $X^{*}, Y^{*}, A, B: X \rightrightarrows X^{*}, M: Y \rightrightarrows Y^{*}$ are multi-valued maximal monotone operators, and $L: X \rightarrow Y$ is linear bounded. Based on the Fitzpatrick function, new characterizations for the maximality of an operator as well as simpler proofs, improvements of previously known results, and several new results on the topic are presented.
\end{abstract}

Mathematics Subject Classification (2000): 47H05, 46N10.

Key words: maximal monotone operator, Fitzpatrick function, sum and chain rules 


\section{Introduction}

The problem concerning the maximality of the sum of two maximal monotone operators was first stated and solved by Rockafellar in reflexive Banach spaces followed by a sum rule for the convex subdifferential in general Banach spaces (see [8, Theorem 1], [15, Theorem 2.8.3]). At the same time the conjecture which states that the reflexivity of the space can be avoided was formulated. Later, the sum rule for full-space domain operators was proved by Heisler (see [10, Theorem 37.4] or Theorem 4.1 below) and for single-valued linear operators by Phelps and Simons (see [6, Theorem 7.2]). Recently, Voisei [11,12,13] proved similar calculus rules for closed convex domain monotone operators in nonreflexive Banach spaces under weaker forms of the qualification constraint and solved completely the linear case (see $[12,13]$ or Corollary 5.14 . below). Using a topological argument, the sum rule for operators with the intersection of their domain interiors non-empty was shown to hold by Borwein (see [1]). Chain rules in the context of reflexive Banach spaces were obtained by Penot [5], Zălinescu [14], and Borwein [1].

In the present note we want to shed a new light on the ideas of the proofs and present new points of view as well as simpler arguments, improvements of some of the past results, and new results concerning the maximality of the sum or of the precomposition with a linear operator in the non-reflexive Banach space setting.

The plan of the paper is as follows. Next section introduces the Fitzpatrick and Penot functions together with their main features. In Section 3 new characterizations for the maximality and representability of an operator are discussed. Section 4 contains a simple proof of Heisler's result. Section 5 deals with the calculus of maximal monotone operators. This paper concludes with some improvements of the results contained in Voisei $[12,13]$ and several other new results on the topic.

\section{The natural dual system. The Fitzpatrick and Penot functions}

Let $X$ be a Banach space with dual $X^{*}$ and bi-dual $X^{* *}$. A multi-valued operator $A: D(A) \subset X \rightrightarrows X^{*}$ is called monotone if, for every $x_{1}, x_{2} \in D(A)$, $x_{1}^{*} \in A x_{1}, x_{2}^{*} \in A x_{2}$

$$
\left\langle x_{1}-x_{2}, x_{1}^{*}-x_{2}^{*}\right\rangle \geq 0
$$

where

$$
p\left(x, x^{*}\right)=\left\langle x, x^{*}\right\rangle=x^{*}(x),\left(x, x^{*}\right) \in X \times X^{*},
$$

stands for the duality pairing in $X \times X^{*}$.

For the sake of notation simplicity we identify operators with their graphs and write

$$
x \in D(A), x^{*} \in A x \Leftrightarrow\left(x, x^{*}\right) \in A .
$$

With this notation $A$ is monotone iff $p\left(a_{1}-a_{2}\right) \geq 0$ for every $a_{1}, a_{2} \in A$. 
A monotone operator is considered maximal monotone if it is maximal in the sense of inclusion in $X \times X^{*}$.

Let $Z=X \times X^{*}$. The natural dual system is formed by $(Z, Z)$ with the dual product

$$
\left(x, x^{*}\right) \cdot\left(y, y^{*}\right)=x^{*}(y)+y^{*}(x), x, y \in X, x^{*}, y^{*} \in X^{*}
$$

The convex conjugate with respect to the natural duality of $f: Z \rightarrow \mathbb{R} \cup\{\infty\}$ is given by

$$
f^{*}(w)=\sup \{w \cdot z-f(z) ; z \in Z\}, w \in Z
$$

Notice that

$$
\begin{gathered}
z \cdot z=2 p(z), p(\lambda z)=\lambda^{2} p(z), \\
p\left(z_{1} \pm z_{2}\right)=p\left(z_{1}\right)+p\left(z_{2}\right) \pm z_{1} \cdot z_{2} \\
p\left(z_{1}+z_{2}\right)+p\left(z_{1}-z_{2}\right)=2\left(p\left(z_{1}\right)+p\left(z_{2}\right)\right),
\end{gathered}
$$

for every $\lambda \in \mathbb{R}, z, z_{1}, z_{2} \in Z$.

On $Z$ we fix a topology compatible with the natural duality such as the strong $\times$ weakly-star topology. In the sequel all topological notions will be understood with respect to this fixed topology on $Z$ if not otherwise specified.

For $\emptyset \neq A \subset Z$ let

$$
p_{A}=p+i_{A},
$$

where $i_{A}(z)=0$, if $z \in A, i_{A}(z)=\infty$, otherwise; is the indicator of $A$.

The Fitzpatrick function of $A$ is $h_{A}: Z \rightarrow \mathbb{R} \cup\{\infty\}$ defined by

$$
h_{A}=p_{A}^{*}
$$

An alternative form for $h_{A}$ is given by

$$
h_{A}(z)=\sup _{a \in A}\{z \cdot a-p(a)\}=p(z)-\inf _{a \in A} p(z-a), z \in Z .
$$

The conjugate of $h_{A}$

$$
\varphi_{A}=h_{A}^{*}=\mathrm{clco} p_{A},
$$

is called the Penot function of $A$ and it represents the greatest proper convex lower semicontinuous function majorized by $p_{A}$.

In the sequel, for $h: Z \rightarrow \mathbb{R} \cup\{\infty\}$, the following set notation will be frequently used

$$
\begin{aligned}
& \{h=p\}=\{z \in Z ; h(z)=p(z)\}, \\
& \{h \geq p\}=\{z \in Z ; h(z) \geq p(z)\}, \\
& \{h \leq p\}=\{z \in Z ; h(z) \leq p(z)\} .
\end{aligned}
$$

PROPOSITION 2.1. For every monotone $A \subset Z$ we have

i) $\varphi_{A}(z) \geq p(z)$, for every $z \in Z$,

ii) $A \subset\left\{\varphi_{A}=p\right\}$, 
iii) $A \subset\left\{h_{A}=p\right\}$,

iv) $D(A) \times X^{*} \subset\left\{h_{A} \geq p\right\}$.

Proof. iv) If $z=\left(x, x^{*}\right) \in D(A) \times X^{*}$ then there exists $\alpha^{*} \in A x$. Let $a=\left(x, \alpha^{*}\right) \in A$. We have $z \cdot a-p(a)=p(z)$. According to (9), this yields $h_{A}(z) \geq p(z)$, i.e., $D(A) \times X^{*} \subset\left\{h_{A} \geq p\right\}$.

iii) Let $z \in A$. Pick $a=z$ in (9) to find $h_{A}(z) \geq p(z)$. Since $A$ is monotone we get $p(z-a)=p(a)-z \cdot a+p(z) \geq 0$, for every $a \in A$. This gives us $h_{A}(z) \leq p(z)$. Therefore $A \subset\left\{h_{A}=p\right\}$ and $h_{A} \leq p_{A}$ in $Z$. Because $h_{A}$ is proper convex lower semicontinuous this yields

$$
h_{A} \leq \varphi_{A} \text { in } Z,
$$

for every $A$ monotone.

Let $\mathcal{A}$ be a maximal monotone extension of $A$. By Theorem 3.3. below, we know that $h_{\mathcal{A}} \geq p$ in $Z$. We have $p_{A} \geq p_{\mathcal{A}}$ and

$$
\varphi_{A} \geq \varphi_{\mathcal{A}} \geq h_{\mathcal{A}} \geq p \text { in } Z
$$

i.e., i) holds.

Subpoint ii) is straight forward from i) and $\varphi_{A} \leq p_{A}$ in $Z$. The proof is complete.

For other properties of $h_{A}, \varphi_{A}$ see [12, Proposition 2].

\section{Representability and maximality}

DEFINITION 3.1. A multi-valued operator $A$ is called representable in $Z=$ $X \times X^{*}$ if there is a proper convex lower semicontinuous $h: Z \rightarrow \mathbb{R} \cup\{\infty\}$ such that

i) $h(z) \geq p(z)$, for every $z \in Z$, i.e., $\{h \geq p\}=Z$,

ii) $z \in A$ iff $h(z)=p(z)$, i.e., $A=\{h=p\}$.

A function $h$ with properties i), ii) is called a representative of $A$. Notice that if $h$ is a representative of $A$ then from $A \subset\{h=p\}$ we get $h \leq p_{A}$ in $Z$ followed by

$$
h \leq \varphi_{A}, h^{*} \geq h_{A} \text { in } Z .
$$

LEMMA 3.1. ([5, Proposition 4]) Every representable operator $A$ is monotone.

Proof. Since $A$ is representable, there exists $h: Z \rightarrow \mathbb{R} \cup\{\infty\}$ such that $h \geq p$ in $Z$ and $z \in A$ iff $h(z)=p(z)$. Therefore, from the convexity of $h$ we get that, for every $z_{1}, z_{2} \in A=\{h=p\}$

$$
\frac{1}{2} p\left(z_{1}\right)+\frac{1}{2} p\left(z_{2}\right)-\frac{1}{4} p\left(z_{1}-z_{2}\right)=p\left(\frac{1}{2} z_{1}+\frac{1}{2} z_{2}\right)
$$




$$
\leq h\left(\frac{1}{2} z_{1}+\frac{1}{2} z_{2}\right) \leq \frac{1}{2} h\left(z_{1}\right)+\frac{1}{2} h\left(z_{2}\right)=\frac{1}{2} p\left(z_{1}\right)+\frac{1}{2} p\left(z_{2}\right),
$$

that is $p\left(z_{1}-z_{2}\right) \geq 0$, for every $z_{1}, z_{2} \in A$.

We prove that an operator is representable iff its Penot function becomes a representative.

THEOREM 3.2. A is representable iff $\varphi_{A}$ is a representative of $A$.

Proof. For the direct implication let $h$ be a representative of $A$. From $A=\{h=p\}$ we know that $h \leq p_{A}$ in $Z$. Therefore

$$
\varphi_{A} \geq h \geq p, \text { in } Z
$$

Combined with $A \subset\left\{\varphi_{A}=p\right\}$ the previous inequality shows that $A=\left\{\varphi_{A}=\right.$ $p$, which implies that $\varphi_{A}$ is a representative of $A$. The converse implication is plain.

For different proofs of the previous result see e.g. [4,5].

The following characterization of maximality in terms of representability is due to Fitzpatrick [2, Theorem 3.8]. For the sake of completeness we provide the reader with a short proof.

THEOREM 3.3. A multi-valued operator $A$ is maximal monotone iff $h_{A}$ is a representative of $A$.

Proof. If $A$ is maximal monotone then for every $z \notin A$ there exists an $a \in A$ such that $p(z-a)<0$. Hence, from (9) we have $h_{A}(z)>p(z)$ for every $z \notin A$. Since $h_{A}(z)=p(z)$, for every $z \in A$ (see Proposition 2.1. iii)) this implies that $h_{A} \geq p$ in $Z$ and $h_{A}(z)=p(z)$ iff $z \in A$, that is $h_{A}$ is a representative of $A$.

Conversely, from Lemma 3.1. or from $h_{A}(z)=p(z)$ for every $z \in A$ and (9) we get $p(z-a) \geq 0$ for every $z, a \in A$, i.e., $A$ is monotone.

Take $z_{0} \in Z$ such that $p\left(z_{0}-a\right) \geq 0$ for every $a \in A$. Again, from (9) we find $h_{A}\left(z_{0}\right)=p\left(z_{0}\right)$, that is $z_{0} \in A$, since $h_{A}$ is a representative of $A$. We showed that $A$ is maximal monotone. The proof is complete.

Clearly, every maximal monotone operator is representable. The question whether the converse holds appears naturally in this context. The following characterization of maximality in terms of representability appeared first in Voisei [11, Theorem 2.3]. For the sake of convenience we provide the reader with a simpler proof.

THEOREM 3.4. $A$ is maximal monotone iff $A$ is representable and $h_{A} \geq p$ in $Z$. 
Proof. The direct implication is trivial since $h_{A}$ is a representative of $A$.

Conversely, we know that $A$ is monotone since it is representable. According to Proposition 2.1., we have $A \subset\left\{h_{A}=p\right\}$. To conclude that $h_{A}$ is a representative of $A$ and consequently that $A$ is maximal monotone, it is enough to prove that $\left\{h_{A}=p\right\} \subset A$. Let $z \in\left\{h_{A}=p\right\}$. Clearly, $z$ is a global minimum point of $h_{A}-p$. Therefore

$$
0 \in \partial\left(h_{A}-p\right)(z),
$$

where " $\partial$ " denotes the Clarke-Rockafellar subdifferential. Since $p$ is continuously Gâteaux differentiable with $\partial(-p(z))=\{-z\}$ and $h_{A}$ is convex, relation (16) reduces to $z \in \partial h_{A}(z)$ which can be equivalently restated as

$$
h_{A}(z)+\varphi_{A}(z)=2 p(z) .
$$

This implies $z \in\left\{\varphi_{A}=p\right\}=A$ because $h_{A}(z)=p(z)$ and $A$ is representable. The proof is complete.

Remark 3.5. Condition $h_{A} \geq p$ in $Z$ is sometimes referred to as $A$ is of negative infimum type or NI in $X \times X^{*}$. Hence the previous characterization theorem can be restated as

$$
\text { Maximal Monotone }=\text { Representable }+ \text { NI }
$$

This characterization of maximality is more versatile because most of the times the representability of operators is easily checked. Usually, the difficulty lies into proving that the operators are of NI type.

\section{A simple proof of Heisler's result}

Previous to the papers $[11,12]$ there are two note-worthy results for the maximality of the sum in a non-reflexive Banach space setting; the result of Heisler for full-space domain operators and the result of Phelps \& Simons (see [6, Theorem 7.2]) for linear single-valued operators. We provide a simpler proof of the Heisler result in order to observe the usefulness of the Fitzpatrick function and mention that in the linear multi-valued case the problem has been completely solved (see $[12,13]$ or Theorem 5.13. below).

Recall the Heisler result

THEOREM 4.1. ([10, Theorem 37.4]) Let $X$ be a Banach space possibly nonreflexive. If $A, B$ are maximal monotone in $X \times X^{*}$ with $D(A)=D(B)=X$ then $A+B$ is maximal monotone.

The previous proof of this result relies on a topological characterization of maximal monotone operators with full-space domain. Our argument is based on the following two lemmas 
LEMMA 4.2. Let $A$ be monotone with $D(A)=X$. Then $A$ is of NI type in $Z=X \times X^{*}$, i.e.,

$$
h_{A}(z) \geq p(z) \text {, for every } z \in Z,
$$

and $\left\{h_{A}=p\right\}$ is the only maximal monotone extension of $A$ in $X \times X^{*}$.

Proof. According to Proposition 2.1. iv), $\left\{h_{A}=p\right\}=Z$, that is, $A$ is (NI). For the second part notice that $\left\{h_{A}=p\right\}$ is representable monotone and every maximal monotone extension $\mathcal{A}$ of $A$ satisfies

$$
\mathcal{A} \subset\left\{h_{A} \leq p\right\}=\left\{h_{A}=p\right\} .
$$

Therefore $\mathcal{A}=\left\{h_{A}=p\right\}$ and $\left\{h_{A}=p\right\}$ is the unique maximal monotone extension of $A$.

LEMMA 4.3. Let $A$ be monotone with $D(A)=X$. Then $A$ is maximal monotone iff $A$ has convex values and $A$ is closed with respect to the strong $\times$ weakly-star convergence of bounded nets in $X \times X^{*}$ given by $\left(x_{\alpha}, x_{\alpha}^{*}\right) \rightarrow\left(x, x^{*}\right) \Leftrightarrow x_{\alpha} \rightarrow x$, strongly in $X, x_{\alpha}^{*} \rightarrow x^{*}$, weakly star in $X^{*}$, and $\left(x_{\alpha}^{*}\right)_{\alpha}$ is bounded in $X^{*}$.

Proof. The direct implication is clear since every maximal monotone operator has convex values and is closed with respect to " $\rightarrow$ ".

For the converse it is enough to show that $\left\{h_{A}=p\right\} \subset A$.

Since $A$ is closed with respect to " $\rightarrow$ " we prove first that $A$ has weakly-star closed values. Indeed, if $\left(x_{\alpha}^{*}\right)_{\alpha} \subset A x, x \in X$, and $x_{\alpha}^{*} \rightarrow x^{*}$ weakly-star in $X^{*}$ then $\left(x_{\alpha}=x, x_{\alpha}^{*}\right) \rightarrow\left(x, x^{*}\right)$ because $A x$ is bounded. Therefore, $x^{*} \in A x$, i.e., $A x$ is weakly-star closed for every $x \in X$.

Let $z=\left(x_{0}, x_{0}^{*}\right) \in\left\{h_{A}=p\right\}$, that is, for every $\left(a, a^{*}\right) \in A$

$$
\left\langle x_{0}-a, x_{0}^{*}-a^{*}\right\rangle \geq 0 \text {. }
$$

Assume by contradiction that $x_{0}^{*} \notin A x_{0}$. By a separation theorem we find $v_{0} \in X$, such that

$$
\left\langle v_{0}, x_{0}^{*}\right\rangle>\sup _{x^{*} \in A x_{0}}\left\langle v_{0}, x^{*}\right\rangle .
$$

For $t>0$, denote by $a_{t}=x_{0}+t v_{0}$ and take $a_{t}^{*} \in A a_{t}$ in (19) to find

$$
\left\langle v_{0}, x_{0}^{*}-a_{t}^{*}\right\rangle \leq 0 .
$$

Notice that for $t \downarrow 0, a_{t} \rightarrow x_{0}$, strongly in $X$. Because $A$ is locally bounded at $x_{0},\left(a_{t}^{*}\right)_{t}$ is bounded in $X^{*}$. Therefore, by the Alaoglu Theorem, at least on a subnet, we have $\left(a_{t}, a_{t}^{*}\right) \rightarrow\left(x_{0}, a_{0}^{*}\right) \in A$.

Pass to limit in (21) with $t \downarrow 0$ to get $\left\langle v_{0}, a_{0}^{*}\right\rangle \geq\left\langle v_{0}, x_{0}^{*}\right\rangle$ which contradicts (201). We proved $z \in A$, that is $A=\left\{h_{A}=p\right\}$. The proof is complete.

Proof of Theorem 4.1. It is straight forward to show that if $A, B$ are closed with respect to " $\rightarrow$ " and have convex values then $A+B$ is " $\rightarrow$ " closed and has 
convex values, because $A, B$ are locally bounded. According to Lemma 4.3., $A+B$ is maximal monotone.

\section{Calculus rules for representable and maximal monotone operators}

Our first concern in this section is the representability of $T:=L^{*} M L$ : $X \rightrightarrows X^{*}$, where $X, Y$ are Banach spaces, $L: X \rightarrow Y$ is linear bounded, and $M: Y \rightrightarrows Y^{*}$ is representable. Let $r_{M}$ be a representative of $M$.

Our choice for a representative of $T$ is $r: X \times X^{*} \rightarrow \overline{\mathbb{R}}$,

$$
r\left(x, x^{*}\right)=\inf \left\{r_{M}\left(L x, y^{*}\right) ; L^{*} y^{*}=x^{*}\right\},\left(x, x^{*}\right) \in X \times X^{*} .
$$

Notice that $r \geq p$ in $Z, T \subset\{r=p\}$, and $T=\{r=p\}$ whenever the "inf" in the definition of $r$ is attained for all $\left(x, x^{*}\right) \in D(r)$. Therefore, it is enough to study conditions which assures that the "inf" in (22) becomes a "min".

For a subset $S$ of a Banach space $X$ we denote by ${ }^{i} S$ the relative algebraic interior of $S$. We define ${ }^{\text {ic }} S={ }^{i} S$ if the affine hull of $S$ is closed and ${ }^{\text {ic }} S=\emptyset$ otherwise.

THEOREM 5.1. Let $X, Y$ be Banach spaces, $L: X \rightarrow Y$ be linear bounded, $M: Y \rightrightarrows Y^{*}$ be representable, and $r_{M}$ be a representative of $M$. If

$$
0 \in{ }^{\text {ic }}\left(R(L)-P_{Y} D\left(r_{M}^{*}\right)\right)
$$

then $T:=L^{*} M L: X \rightrightarrows X^{*}$ is representable. Here $P_{Y}: Y \times Y^{*} \rightarrow Y$, $P_{Y}\left(y, y^{*}\right)=y,\left(y, y^{*}\right) \in Y \times Y^{*}$ is the projection of $Y \times Y^{*}$ onto $Y, L^{*}: Y^{*} \rightarrow$ $X^{*}$ denotes the adjoint of $L, R(L)$ is the range of $L$, and $r_{M}^{*}$ stands for the convex conjugate with respect to the natural duality.

$$
\begin{aligned}
& \text { Proof. Consider } \varphi: X \times X^{*} \rightarrow \overline{\mathbb{R}}, \\
& \qquad \varphi\left(x, x^{*}\right)=\inf \left\{r_{M}^{*}\left(L x, y^{*}\right) ; L^{*} y^{*}=x^{*}\right\}, \quad\left(x, x^{*}\right) \in X \times X^{*} .
\end{aligned}
$$

Since $\varphi\left(x, x^{*}\right)=\inf \left\{r_{M}^{*}\left(y, y^{*}\right) ;\left(y, y^{*}\right) \in C\left(x, x^{*}\right)\right\}$, where the process $C \subset$ $X \times X^{*} \times Y \times Y^{*}$ is defined by

$$
\left(x, x^{*}, y, y^{*}\right) \in C \text { iff } y=L x, x^{*}=L^{*} y^{*},
$$

$R(C)=R(L) \times Y^{*}$, and condition $0 \in{ }^{\text {ic }}\left(R(L)-P_{Y} D\left(r_{M}^{*}\right)\right)$ is equivalent to $0 \in{ }^{\text {ic }}\left(R(C)-D\left(r_{M}^{*}\right)\right)$, according to [15, T 2.8.6], we may apply the chain rule to get

$\varphi_{Z^{*}}^{*}\left(x^{*}, x^{* *}\right)=\min \left\{r_{M}^{* *}\left(y^{*}, y^{* *}\right) ;\left(x^{*}, x^{* *}\right) \in C^{*}\left(y^{*}, y^{* *}\right)\right\},\left(x^{*}, x^{* *}\right) \in X^{*} \times X^{* *}$.

Here $\varphi_{Z^{*}}^{*}$ denotes the convex conjugate of $\varphi$ in $Z^{*}=X^{*} \times X^{* *}$ and is weaklystar lower semicontinuous in $Z^{*}$ which makes $\varphi^{*}=\varphi_{Z^{*}}^{*} / Z$ weakly $\times$ weakly-star lower semi-continuous in $Z$. 
The adjoint of $C$ is given by

$$
\left(y^{*}, y^{* *}, x^{*}, x^{* *}\right) \in C^{*} \text { iff } y^{* *}=L^{* *} x^{* *}, x^{*}=L^{*} y^{*} .
$$

By the bi-conjugate formula for $x^{* *}=x \in X$ relation (26) becomes

$$
r\left(x, x^{*}\right)=\varphi^{*}\left(x, x^{*}\right)=\min \left\{r_{M}\left(L x, y^{*}\right) ; L^{*} y^{*}=x^{*}\right\},\left(x, x^{*}\right) \in X \times X^{*} .
$$

Relation (28) shows that $r$ is a representative of $T$ and consequently $T$ is representable.

PROPOSITION 5.2. Let $X$ be a Banach space, $L: X \rightarrow X \times X, L x=(x, x)$, $x \in X$, and $U, V$ be convex subsets of $X$. Then

$$
\begin{gathered}
0 \in{ }^{i}(R(L)-U \times V) \Leftrightarrow 0 \in{ }^{i}(U-V) . \\
0 \in{ }^{\mathrm{ic}}(R(L)-U \times V) \Leftrightarrow 0 \in{ }^{\mathrm{ic}}(U-V) .
\end{gathered}
$$

Proof. Consider the "difference function" $\mathcal{D}: X \times X \rightarrow X$,

$$
\mathcal{D}\left(x_{1}, x_{2}\right)=x_{2}-x_{1}, x_{1}, x_{2} \in X .
$$

Notice that $\operatorname{Ker} \mathcal{D}=R(L)$, and let "aff" denote the affine hull of a subset in $X$ or $X \times X$. We have

$$
\begin{gathered}
F:=\operatorname{aff}(R(L)-U \times V)=R(L)-\operatorname{aff} U \times \operatorname{aff} V, \\
\mathcal{D}(F)=\operatorname{aff}(U-V) .
\end{gathered}
$$

According to [15, Corollary 1.3.15] $\mathcal{D}(F)$ is closed iff $F=F+\operatorname{Ker} \mathcal{D}$ is closed. Therefore, $\operatorname{aff}(R(L)-U \times V)$ is closed iff $\operatorname{aff}(U-V)$ is closed.

Since $U, V$ are convex, condition $0 \in{ }^{i}(R(L)-U \times V)$ is equivalent to $\bigcup n(R(L)-U \times V)$ is a linear subspace and $0 \in{ }^{i}(U-V)$ is equivalent to $n \geq 1$

$\bigcup_{n \geq 1} n(U-V)$ is a linear subspace (see $[15,(1.1)]$ ).

But

$$
\left(x_{1}, x_{2}\right) \in R(L)-U \times V \text { iff } \mathcal{D}\left(x_{1}, x_{2}\right)=x_{2}-x_{1} \in U-V,
$$

which shows that $\bigcup_{n \geq 1} n(R(L)-U \times V)$ is a linear subspace iff $\bigcup_{n \geq 1} n(U-V)$ is a linear subspace. Hence $0 \in{ }^{i}(R(L)-U \times V)$ iff $0 \in{ }^{i}(U-V)$. The proof is complete.

For a generalization of (29) see [16, Proposition 2.1]. 
THEOREM 5.3. Let $X$ be a Banach space and $A, B: X \rightrightarrows X^{*}$ be representable with

$$
0 \in{ }^{\text {ic }}\left(P_{X} D\left(r_{A}^{*}\right)-P_{X} D\left(r_{B}^{*}\right)\right),
$$

where $r_{A}, r_{B}$ are representatives of $A, B$ and $P_{X}$ stands for the projection of $X \times X^{*}$ onto $X$. Then $A+B$ is representable.

Proof. First argument. We apply Theorem 5.1. for $X, Y=X \times X, L x=$ $(x, x), x \in X$, and $M\left(x_{1}, x_{2}\right)=A x_{1} \times B x_{2},\left(x_{1}, x_{2}\right) \in D(M)=D(A) \times D(B)$ for which $L^{*} M L=A+B$,

$$
r_{M}\left(x_{1}, x_{2}, x_{1}^{*}, x_{2}^{*}\right)=r_{A}\left(x_{1}, x_{1}^{*}\right)+r_{B}\left(x_{2}, x_{2}^{*}\right), x_{1}, x_{2} \in X, x_{1}^{*}, x_{2}^{*} \in X^{*},
$$

is a representative of $M$ with

$$
r_{M}^{*}\left(x_{1}, x_{2}, x_{1}^{*}, x_{2}^{*}\right)=r_{A}^{*}\left(x_{1}, x_{1}^{*}\right)+r_{B}^{*}\left(x_{2}, x_{2}^{*}\right), x_{1}, x_{2} \in X, x_{1}^{*}, x_{2}^{*} \in X^{*},
$$

$P_{Y} D\left(r_{M}^{*}\right)=P_{X} D\left(r_{A}^{*}\right) \times P_{X} D\left(r_{B}^{*}\right)$, and according to Proposition 5.2., condition $0 \in{ }^{\text {ic }}\left(P_{X} D\left(r_{A}^{*}\right)-P_{X} D\left(r_{B}^{*}\right)\right)$ is equivalent to $0 \in{ }^{\text {ic }}\left(R(L)-P_{Y} D\left(r_{M}^{*}\right)\right)$.

Second argument. Let $\left(x_{0}, x_{0}^{*}\right) \in\left\{\varphi_{A+B}=p\right\}$. Since $\varphi_{A+B}=h_{A+B}^{*}$ we have that for every $\left(u, u^{*}\right) \in Z$

$$
h_{A+B}\left(u, u^{*}\right)-\left\langle u, x_{0}^{*}\right\rangle-\left\langle x_{0}, u^{*}\right\rangle+\left\langle x_{0}, x_{0}^{*}\right\rangle \geq 0 .
$$

Let $\mathcal{X}=X \times X^{*} \times X^{*}, \mathcal{Y}=X$ and consider the function $\Phi: \mathcal{X} \times \mathcal{Y} \rightarrow \mathbb{R} \cup\{\infty\}$ given by

$$
\Phi\left(x, x^{*}, z^{*} ; y\right)=r_{A}^{*}\left(x+y, x^{*}\right)+r_{B}^{*}\left(x, z^{*}\right)-\left\langle x, x_{0}^{*}\right\rangle-\left\langle x_{0}, x^{*}+z^{*}\right\rangle+\left\langle x_{0}, x_{0}^{*}\right\rangle,
$$

$x, y \in X, x^{*}, z^{*} \in X^{*}$.

Notice that, since $r_{A}^{*} \geq h_{A}, r_{B}^{*} \geq h_{B}$ (see (13)), we get

$$
\begin{gathered}
\Phi\left(x, x^{*}, z^{*} ; 0\right) \geq h_{A}\left(x, x^{*}\right)+h_{B}\left(x, z^{*}\right)-\left\langle x, x_{0}^{*}\right\rangle-\left\langle x_{0}, x^{*}+z^{*}\right\rangle+\left\langle x_{0}, x_{0}^{*}\right\rangle \\
\geq h_{A+B}\left(x, x^{*}+z^{*}\right)-\left\langle x, x_{0}^{*}\right\rangle-\left\langle x_{0}, x^{*}+z^{*}\right\rangle+\left\langle x_{0}, x_{0}^{*}\right\rangle \geq 0,
\end{gathered}
$$

for every $\left(x, x^{*}, z^{*}\right) \in \mathcal{X}$, i.e., $\inf _{\chi \in \mathcal{X}} \Phi(\chi, 0) \geq 0$.

If $P_{\mathcal{Y}}(\chi, y)=y,(\chi, y) \in \mathcal{X} \times \mathcal{Y}$, is the projection of $\mathcal{X} \times \mathcal{Y}$ onto $\mathcal{Y}$ then $P_{\mathcal{Y}} D(\Phi)=P_{X} D\left(r_{A}^{*}\right)-P_{X} D\left(r_{B}^{*}\right)$ and condition (34) spells $0 \in{ }^{\text {ic }}\left(P_{\mathcal{Y}} D(\Phi)\right)$.

This allows us to apply the fundamental duality formula (see e.g. [15, Theorem 2.7.1 (vii)]) to get

$$
\inf _{\chi \in \mathcal{X}} \Phi(\chi, 0)=\max _{y^{*} \in Y^{*}=X^{*}}\left(-\Phi^{*}\left(0, y^{*}\right)\right) \geq 0 .
$$

Therefore, there exists $y^{*} \in X^{*}$ such that

$$
\Phi^{*}\left(0, y^{*}\right)=\sup \left\{\left\langle y, y^{*}\right\rangle-\Phi\left(x, x^{*}, z^{*} ; y\right) ; x, y \in X, x^{*}, z^{*} \in X^{*}\right\} \leq 0,
$$


that is

$$
r_{A}^{*}\left(x+y, x^{*}\right)+r_{B}^{*}\left(x, z^{*}\right)-\left\langle x, x_{0}^{*}\right\rangle-\left\langle x_{0}, x^{*}+z^{*}\right\rangle+\left\langle x_{0}, x_{0}^{*}\right\rangle-\left\langle y, y^{*}\right\rangle \geq 0,
$$

for every $x, y \in X, x^{*}, z^{*} \in X^{*}$.

Using the substitution $x+y=z$, we find

$$
r_{A}^{*}\left(z, x^{*}\right)+r_{B}^{*}\left(x, z^{*}\right)-\left\langle x, x_{0}^{*}\right\rangle-\left\langle x_{0}, x^{*}+z^{*}\right\rangle+\left\langle x_{0}, x_{0}^{*}\right\rangle-\left\langle z-x, y^{*}\right\rangle \geq 0,
$$

for every $x, y \in X, x^{*}, z^{*} \in X^{*}$, or

$$
\left[\left\langle z, y^{*}\right\rangle+\left\langle x_{0}, x^{*}\right\rangle-r_{A}^{*}\left(z, x^{*}\right)\right]+\left[\left\langle x, x_{0}^{*}-y^{*}\right\rangle+\left\langle x_{0}, z^{*}\right\rangle-r_{B}^{*}\left(x, z^{*}\right)\right] \leq\left\langle x_{0}, x_{0}^{*}\right\rangle,
$$

for every $x, y \in X, x^{*}, z^{*} \in X^{*}$, that is

$$
r_{A}\left(x_{0}, y^{*}\right)+r_{B}\left(x_{0}, x_{0}^{*}-y^{*}\right) \leq\left\langle x_{0}, x_{0}^{*}\right\rangle .
$$

Because $r_{A}, r_{B}$ are representatives of $A, B$ relation (42) is equivalent to

$$
r_{A}\left(x_{0}, y^{*}\right)=\left\langle x_{0}, y^{*}\right\rangle, r_{B}\left(x_{0}, x_{0}^{*}-y^{*}\right)=\left\langle x_{0}, x_{0}^{*}-y^{*}\right\rangle,
$$

that is, $\left(x_{0}, y^{*}\right) \in A,\left(x_{0}, x_{0}^{*}-y^{*}\right) \in B$. Hence $\left(x_{0}, x_{0}^{*}\right) \in A+B$. We proved that $\left\{\varphi_{A+B}=p\right\} \subset A+B$ and this is enough in order to conclude that $A+B$ is representable.

Remark 5.4. The typical example of a representative of $A$ is provided by the Penot function $\varphi_{A}$. Therefore, in a particular case, Theorems 5.1, 5.3 can be restated as

COROLLARY 5.5. Let $X, Y$ be two Banach spaces, $L: X \rightarrow Y$ be linear bounded, and $M: Y \rightrightarrows Y^{*}$ be representable. If

$$
0 \in{ }^{\mathrm{ic}}\left(R(L)-P_{Y} D\left(h_{M}\right)\right)
$$

then $T:=L^{*} M L: X \rightrightarrows X^{*}$ is representable.

COROLLARY 5.6. Let $X$ be a Banach space and $A, B: X \rightrightarrows X^{*}$ be representable with

$$
0 \in{ }^{\mathrm{ic}}\left(P_{X} D\left(h_{A}\right)-P_{X} D\left(h_{B}\right)\right) .
$$

Then $A+B$ is representable.

PROPOSITION 5.7. If $M: Y \rightrightarrows Y^{*}$ is monotone in the Banach space $Y$, $D(M)$ is closed convex, and

$$
M=M+N_{D(M)}
$$

then $M$ is of $N I$ type and $D(M)=P_{Y} D\left(h_{M}\right)$. Here $N_{D(M)}$ stands for the convex normal cone to $D(M)$. 
Proof. For every $y \in D(M)$ there is $y^{*} \in Y^{*}$ such that $\left(y, y^{*}\right) \in M \subset\left\{h_{M}=\right.$ $p\} \subset D\left(h_{M}\right)$, that is, $D(M) \subset P_{Y} D\left(h_{M}\right)$.

Conversely, let $y \in P_{Y} D\left(h_{M}\right)$, that is, $h_{M}\left(y, y^{*}\right)<\infty$, for some $y^{*} \in Y^{*}$. Hence, for every $\left(m, m^{*}\right) \in M$, we have

$$
\left\langle y-m, m^{*}\right\rangle+\left\langle m, y^{*}\right\rangle \leq C<\infty .
$$

From (45), (46) and because $N_{D(M)}(y)$ is a cone for every $y \in D(M)$, we get

$$
t\left\langle y-m, n^{*}\right\rangle+\left\langle y-m, m^{*}\right\rangle+\left\langle m, y^{*}\right\rangle \leq C<\infty,
$$

for every $t>0, m \in D(M), m^{*} \in M m, n^{*} \in N_{D(M)}(m)$.

From (47) it yields that $\left\langle y-m, n^{*}\right\rangle \leq 0$, for every $m \in D(M), n^{*} \in$ $N_{D(M)}(m)$, i.e., $(y, 0)$ is monotonically related to the graph of the maximal monotone operator $N_{D(M)}$. Therefore, $(y, 0) \in N_{D(M)}$, that is, $y \in D(M)$. We proved $P_{Y} D\left(h_{M}\right)=D(M)$, i.e., $D\left(h_{M}\right) \subset D(M) \times X^{*}$. According to Proposition 2.1. iv) this implies $D\left(h_{M}\right) \subset\left\{h_{M} \geq p\right\}$, that is $M$ is NI.

Remark 5.8. Condition (45) is satisfied whenever $M$ is maximal monotone, since $N_{D(M)}$ is monotone, $0 \in N_{D(M)}(y)$ for every $y \in D(M)$, and $M \subset M+$ $N_{D(M)}$. Therefore, every maximal monotone $M$ with $D(M)$ closed convex has $P_{Y} D\left(h_{M}\right)=D(M)$.

THEOREM 5.9. Let $X, Y$ be Banach spaces, $L: X \rightarrow Y$ be linear bounded, $M: Y \rightrightarrows Y^{*}$ be maximal monotone, and $T:=L^{*} M L: X \rightrightarrows X^{*}$.

$(\alpha)$ If $D(T)$ is closed convex and

$$
N_{D(T)}=L^{*} N_{D(M)} L
$$

then $T$ is of NI type.

( $\beta$ ) If $D(M)$ is closed convex and

$$
0 \in{ }^{\text {ic }}(R(L)-D(M))
$$

then $T$ is maximal monotone.

$(\gamma)$ If $D(T)$ is closed and $R(L) \cap \operatorname{int} D(M) \neq \emptyset$ then $T$ is maximal monotone.

Proof. $(\alpha)$ Since $M$ is maximal monotone we know that $M=M+N_{D(M)}$. We find $M(L x)=M(L x)+N_{D(M)}(L x)$ and

$$
T x=L^{*} M(L x)=L^{*} M(L x)+L^{*} N_{D(M)}(L x)=T x+N_{D(T)}(x),
$$

for every $x \in D(T)=L^{-1}(D(M))$, that is, $T=T+N_{D(T)}$ and, according to Proposition 5.7., $T$ is of NI type. 
( $\beta$ ) Since $D(M)$ is closed convex, $D(T)=L^{-1}(D(M))$ is closed convex, $D(M)=P_{Y} D\left(h_{M}\right)$, and (49) becomes (44), and so, by Corollary 5.5., $T$ is representable. Also,

$$
i_{D(T)}(x)=\inf \left\{i_{D(M)}(y) ; y=L x\right\}, x \in D(T) .
$$

Taking into account (49), we may apply the chain rule [15, Theorem 2.8.6 (v)] to get that

$$
N_{D(T)}=L^{*} N_{D(M)} L .
$$

According to $(\alpha), T$ is of NI type. Hence $T$ is maximal monotone.

$(\gamma)$ Because $M$ is maximal monotone with $\operatorname{int} D(M) \neq \emptyset, \operatorname{int} D(M), \overline{D(M)}$ are convex, $\operatorname{int} D(M)=\operatorname{int} \overline{D(M)}, \overline{D(M)}=\overline{\operatorname{int} D(M)}$ (see e.g [10, Theorem 18.4]), $\operatorname{int} D(M)=\operatorname{int} P_{Y} D\left(h_{M}\right)$ (see e.g. [9, Theorem 2.2.]), $R(L)-P_{Y} D\left(h_{M}\right)$ contains 0 in its interior, and (44) follows making $T$ representable.

We prove that

$$
D(T)=L^{-1}(\overline{D(M)}) .
$$

The direct inclusion is plain since $L$ is continuous and $D(T)$ is closed.

Conversely, let $x_{0} \in L^{-1}(\overline{D(M)})$, that is, $L x_{0} \in \overline{D(M)}$. Without loss of generality we may assume that $0 \in \operatorname{int} D(M)$ and $0 \in M 0$. Then $\lambda L x_{0} \in D(M)$, for every $0 \leq \lambda<1$ (see e.g. [15, Theorem 1.1.2]), i.e., $\lambda x_{0} \in D(T)$, for $0 \leq \lambda<1$. Letting $\lambda \uparrow 1$, we find $x_{0} \in \overline{D(T)}=D(T)$.

Relation (52) shows that $D(T)$ is closed convex.

Again, from the chain rule [15, Theorem 2.8.6 (iii)] applied for

$$
i_{D(T)}(x)=i_{L^{-1}(\overline{D(M)})}(x)=\inf \left\{i_{\overline{D(M)}}(y) ; y=L x\right\}, x \in X .
$$

we get (48), that is $T$ is NI and this is sufficient in order to conclude.

THEOREM 5.10. Let $A, B$ be maximal monotone operators in the Banach space $X$.

( $\alpha$ ) If $D(A) \cap D(B)$ is closed convex and

$$
N_{D(A) \cap D(B)}=N_{D(A)}+N_{D(B)},
$$

then $A+B$ is of NI type.

$(\beta)$ If $D(A), D(B)$ are closed convex and

$$
0 \in{ }^{\text {ic }}(D(A)-D(B)),
$$

then $A+B$ is maximal monotone,

$(\gamma)$ If $D(A) \cap D(B)$ is closed, $\overline{D(A)}$ is convex, and $D(A) \cap \operatorname{int} D(B) \neq \emptyset$ then $\overline{D(A)} \cap \overline{D(B)}=D(A) \cap D(B)$ and $A+B$ is maximal monotone.

( $\delta$ ) If $D(A)$ is closed convex and $D(A) \subset D(B)$ then $A+B$ is of NI type.

( $\epsilon)$ If $D(A)$ is closed convex, $D(A) \subset D(B)$, and $0 \in{ }^{\text {ic }}\left(D(A)-P_{X} D\left(h_{B}\right)\right)$ then $A+B$ is maximal monotone. 
Proof. Sub-points $(\alpha),(\beta)$ are direct consequences of Theorem 5.9. $(\alpha),(\beta)$ applied for $Y=X \times X, L x=(x, x), x \in X, L^{*}: Y^{*}=X^{*} \times X^{*} \rightarrow X^{*}$, $L^{*}\left(x^{*}, y^{*}\right)=x^{*}+y^{*}, x^{*}, y^{*} \in X^{*}, M\left(x_{1}, x_{2}\right)=A x_{1} \times B x_{2},\left(x_{1}, x_{2}\right) \in D(M)=$ $D(A) \times D(B)$, for which $L^{*} M L=A+B$. More precisely, subpoint $(\beta)$ follows from Theorem 5.9. $(\beta)$ since $0 \in{ }^{\text {ic }}(D(A)-D(B))$ iff $0 \in{ }^{\text {ic }}(R(L)-D(M))$ (see (30) ). For an alternative proof of $(\beta)$ see [12, Theorem 2].

$(\gamma)$ Without loss of generality assume that $0 \in D(A) \cap \operatorname{int} D(B)$. If $x \in$ $\overline{D(A)} \cap \overline{D(B)}$, then, for every $0 \leq \lambda<1, \lambda x \in \overline{D(A)} \cap \operatorname{int} D(B) \subset \overline{D(A) \cap D(B)}$. Let $\lambda \uparrow 1$ to find $x \in \overline{D(A) \cap D(B)}=D(A) \cap D(B)$, that is $\overline{D(A)} \cap \overline{D(B)}=$ $D(A) \cap D(B)$ and consequently $D(A) \cap D(B)$ is convex.

Therefore, for every $x \in D(A) \cap D(B)$

$$
\begin{gathered}
N_{D(A) \cap D(B)}(x)=N_{\overline{D(A)} \cap \overline{D(B)}}(x) \\
=N_{\overline{D(A)}}(x)+N_{\overline{D(B)}}(x)=N_{D(A)}(x)+N_{D(B)}(x),
\end{gathered}
$$

i.e., (53) holds. The NI type follows from $(\alpha)$ while the representability is a consequence of $D(A) \cap \operatorname{int} D(B) \neq \emptyset$ and Corollary 5.6.

$(\delta)$ Clearly, $D(A) \cap D(B)=D(A)$ is closed convex and since $N_{D(A)}$ is maximal monotone we get

$$
N_{D(A) \cap D(B)}=N_{D(A)}=N_{D(A)}+N_{\overline{c o} D(B)}=N_{D(A)}+N_{D(B)},
$$

i.e., according to $(\alpha), A+B$ is NI. Here " $\overrightarrow{c o}$ " stands for the closed convex hull.

$(\epsilon)$ Condition $0 \in{ }^{\text {ic }}\left(D(A)-P_{X} D\left(h_{B}\right)\right)$ implies the representability of $A+B$. From $(\delta)$ we know that $A+B$ is NI, therefore $A+B$ is maximal monotone.

Remark 5.11. A recent results of Groh [3, Theorem 1.6] is a particular case of our subpoint $(\epsilon)$, for $A$ being a subdifferential and $B$ having a non-empty interior.

The following result of Bauschke presents a different perspective on the subject.

THEOREM 5.12. ([10, Theorem 39.1]) Let $A$ be maximal monotone in the Banach space $X$ and $B: X \rightarrow X^{*}$ be linear with $\langle B x, x\rangle=0$, for every $x \in X$. Then $A+B$ is maximal monotone.

Proof. It is easily checked that for every $\left(x, x^{*}\right) \in X \times X^{*}$

$$
h_{A+B}\left(x, x^{*}\right)=h_{A}\left(x, x^{*}+B^{*} x\right)=h_{A}\left(x, x^{*}-B x\right),
$$

where $B^{*}=-B$ stands for the adjoint of $B$. This equality suffices in order to conclude that $h_{A+B}$ is a representative of $A+B$ and $A+B$ is maximal monotone. 
Notice that under the assumptions of Bauschke's result we have

$$
h_{A+B}\left(x, x^{*}\right)=\inf \left\{h_{A}\left(x, y^{*}\right)+h_{B}\left(x, z^{*}\right) ; y^{*}+z^{*}=x^{*}\right\}=\left(h_{A} \square_{2} h_{B}\right)\left(x, x^{*}\right) \text {, }
$$

for every $\left(x, x^{*}\right) \in X \times X^{*}$, since $h_{B}\left(x, z^{*}\right)=0$, iff $z^{*}=-B^{*} x, h_{B}\left(x, z^{*}\right)=+\infty$, otherwise; where " $\square_{2}$ " denotes the infimal convolution with respect to the second variable.

It is worth noticing that equality (56) assures that $A+B$ is NI and that $A+B$ is maximal monotone whenever the infimal convolution in (56) is exact. Unfortunately, in general (56) does not hold even under the assumptions $D(A)=$ $D(B)=X$ and $X$ is a Hilbert space (see e.g. [7, Example 1]). Other cases in which an equality of type (56) holds are given in the following theorem.

THEOREM 5.13. Let $X, Y$ be Banach spaces.

( $\alpha$ ) If $L: X \rightarrow Y$ is linear bounded, and $M: Y \rightrightarrows Y^{*}$ is maximal monotone with $\operatorname{Graph}(M)$ convex in $X \times X^{*}$ and

$$
0 \in{ }^{\text {ic }}(R(L)-D(M)),
$$

then $T:=L^{*} M L: X \rightrightarrows X^{*}$ is maximal monotone.

$(\beta)$ If $A, B$ are maximal monotone operators in $X$ with $\operatorname{Graph}(A), \operatorname{Graph}(B)$ convex and

$$
0 \in{ }^{\text {ic }}(D(A)-D(B))
$$

then $A+B$ is maximal monotone.

Proof. $(\alpha)$ We have

$$
\begin{aligned}
& p_{T}\left(x, x^{*}\right)=\min \left\{p_{M}\left(L x, y^{*}\right) ; L^{*} y^{*}=x^{*}\right\} \\
& \quad=\min \left\{p_{M}\left(y, y^{*}\right) ;\left(y, y^{*}\right) \in C\left(x, x^{*}\right)\right\},\left(x, x^{*}\right) \in X \times X^{*},
\end{aligned}
$$

where $C \subset X \times X^{*} \times Y \times Y^{*}$ is defined in (25) with adjoint $C^{*}$ given by (27).

Notice that $R(C)=R(L) \times Y^{*}, D\left(p_{M}\right)=M, R(C)-D\left(p_{M}\right)=(R(L)-$ $D(M)) \times Y^{*}$ and condition $0 \in{ }^{\text {ic }}\left(R(C)-D\left(p_{M}\right)\right)$ is equivalent to (57). Moreover, Graph $M$ strongly closed and convex in $X \times X^{*}$ makes $p_{M}$ proper convex strongly lower semicontinuous in $X \times X^{*}$.

We apply the chain rule [15, Theorem 2.8.6 (v)] to get

$$
p_{T}^{*}\left(x^{*}, x^{* *}\right)=\min \left\{p_{M}^{*}\left(y^{*}, y^{* *}\right) ;\left(x^{*}, x^{*}\right) \in C^{*}\left(y^{*}, y^{* *}\right)\right\},
$$

$\left(x, x^{*}\right) \in X \times X^{*}$. For $x^{* *}=x \in X$ we find

$$
h_{T}\left(x, x^{*}\right)=\min \left\{h_{M}\left(L x, y^{*}\right) ; L^{*} y^{*}=x^{*}\right\},
$$

which implies that $h_{T}$ is a representative of $T$, i.e., $T$ is maximal monotone.

( $\beta$ ) Again, take $Y=X \times X, L x=(x, x), x \in X, L^{*}: Y^{*}=X^{*} \times X^{*} \rightarrow X^{*}$, $L^{*}\left(x^{*}, y^{*}\right)=x^{*}+y^{*}, x^{*}, y^{*} \in X^{*}$, and $M\left(x_{1}, x_{2}\right)=A x_{1} \times B x_{2},\left(x_{1}, x_{2}\right) \in$ $D(M)=D(A) \times D(B)$ or Graph $M=\operatorname{Graph} A \times \operatorname{Graph} B$. 
Then $L^{*} M L=A+B$ is maximal monotone by the conclusion of $(\alpha)$, taking into consideration that, in this case, (58) is equivalent to (57).

COROLLARY 5.14. Let $X, Y$ be Banach spaces.

( $\alpha$ ) If $L: X \rightarrow Y$ is linear bounded, and $M: Y \rightrightarrows Y^{*}$ is linear maximal monotone with $R(L)-D(M)$ closed in $Y$, then $T:=L^{*} M L: X \rightrightarrows X^{*}$ is maximal monotone.

$(\beta)$ If $A, B$ are linear maximal monotone with $D(A)-D(B)$ closed in $X$ then $A+B$ is maximal monotone.

Proof. Condition (57) is equivalent to $R(L)-D(M)$ closed in $Y$, since $R(L)-D(M)$ is a subspace. Similarly, (58) becomes $D(A)-D(B)$ is closed in $X$. For a different proof of $(\beta)$ see [13].

It is worth mentioning that in the linear case the qualification constraints contained in $(\alpha),(\beta)$ cannot be further relaxed (see e.g. [10] for a counterexample).

\section{Acknowledgments}

The author would like to thank Dr. C. Zălinescu for several interesting comments made on a preliminary version of this paper.

\section{References}

1. Borwein, J.: Maximal monotonicity via convex analysis, Journal of Convex Analysis 13/14 (2006).

2. Fitzpatrick, S.: Representing monotone operators by convex functions, Workshop/Miniconference on Functional Analysis and Optimization (Canberra, 1988), in: Proc. Centre Math. Anal. Austral. Nat. Univ. 20, Austral. Nat. Univ., Canberra, 1988, pp. 59-65.

3. Groh K.: On monotone operators and forms, Journal of Convex Analysis 12(2) (2005), 417-429.

4. Martinez-Legaz, J.E. and Svaiter, B.F.: Monotone operators representable by l.s.c. convex functions, Set-Valued Analysis 13 (2005), 21-46.

5. Penot, J.-P,: The relevance of convex analysis for the study of monotonicity, (English. English summary) Nonlinear Anal. 58(7-8) (2004), $855-871$. 
6. Phelps, R.R. and Simons S.: Unbounded linear monotone operators on nonreflexive Banach spaces (English. English summary), Journal of Convex Analysis 5(2) (1998), 303-328.

7. Penot, J.-P and Zălinescu, C.: Some problems about the representation of monotone operators by convex functions, Anziam J. 47(1) (2005), 1-20.

8. Rockafellar, R.T.: On the maximality of sums of nonlinear monotone operators, Trans. Amer. Math. Soc. 149 (1970), 75-88.

9. Simons, S.: Dualized and scaled Fitzpatrick functions, Proc. A.M.S. 134(10) (2006), 2983-2987.

10. Simons, S.: Minimax and monotonicity, Lecture Notes in Mathematics, 1693. Springer-Verlag, Berlin, 1998.

11. Voisei, M.D.: A maximality theorem for the sum of maximal monotone operators in non-reflexive Banach Spaces, Math. Sci. Res. J., 10(2) (2006), 36-41.

12. Voisei, M.D.: Calculus rules for maximal monotone operators in general Banach spaces, preprint 2006.

13. Voisei, M.D.: The sum theorem for linear maximal monotone operators, Math. Sci. Res. J. 10(4) (2006), 83-85.

14. Zălinescu C.: A new proof of the maximal monotonicity of the sum using the Fitzpatrick function, Variational analysis and applications, in: Nonconvex Optim. Appl., 79, Springer, New York, 2005, pp. 1159-1172.

15. Zălinescu C.: Convex analysis in general vector spaces, World Scientific Publishing Co., Inc., River Edge, NJ, 2002.

16. Zălinescu C.: On convex sets in general position, Linear Algebra Appl. 64 (1985), 191-198. 